\title{
MaxHopCount: A New Drop Policy to Optimize Messages Delivery Rate in Delay Tolerant Networks
}

\author{
Youssef Harrati, Abdelmounaim Abdali
}

\author{
Cadi Ayyad University, Faculty of Sciences \& Techniques. Laboratoire de Mathématiques Appliquées et Informatique \\ (LAMAI), Marrakech, Morocco
}

\begin{abstract}
Communication has become a necessity, not only between every point on the earth, but also on the globe. That includes hard topography, highlands, underwater areas, and also spacecrafts on other planets. However, the classic wired internet cannot be implemented in such areas, hence, researchers have invented wireless networks. The big challenge for wireless networking nowadays, is maintaining nodes connected in some difficult conditions, such as intermittent connectivity, power failure, and lot of obstacles for the interplanetary networks. In these challenging circumstances, a new networking model arises; it is Delay Tolerant networking which is based on the Store-Carry-and-Forward mechanism. Thus, a node may keep a message in its buffer for long periods of time; until a delivery or forward chance arises then it transmit it to other nodes. One of the big issues that confront this mechanism is the congestion of nodes buffer due to the big number of messages and the limited buffer size. Here, researchers have proposed buffer management algorithms in order to deal with the buffer overload problem, and they called it Drop Policies. In our present work, we propose a new Drop policy which we have compared to other existing policies in different conditions and with different routing protocols, and it always shows good result in term of number of delivered messages, network overhead and also average of latency.
\end{abstract}

Keywords - Buffer Management, Delay Tolerant Networks, Drop Policy, Mobility Models, Network Simulator (ONE), Scheduling.

\section{INTRODUCTION}

$\mathrm{D}$ LAY Tolerant Networks (DTN) is a new concept of networking, which was proposed by Kevin Fall et .al [1] in 2003. Several recent studies are focused on DTN networks, and consider it to be one of the aspects of mobile network development in the future.

The classic TCP-IP [2] Internet model provides an end-to-end communication, which requires a safe path between source and destination, and a large bandwidth. Nevertheless, when a wireless mobile network suffers from the lack of path between source and destination, intermittent connectivity, as well as long latency and limited bandwidth, the TCP concept can, unfortunately, no longer be applied. Under these particular circumstances, DTN networks were introduced.

Every DTN node has a local storage area where it stores messages until a delivery opportunity arises. However, when the node buffer is full of messages (we call it a congested node) and a new message arrives, we don't know what to do and which message is the best to drop to free space without decreasing the delivery rate. Thus, researchers have proposed a set of drop policies.

In this paper, we compare some existing buffer management policies with existing routing protocols, then after deciding which routing protocol and which buffer policies are optimal for such environment,

we will compare these optimal algorithms with our new algorithm "MaxHopCount" which we have developed in our laboratory.

The rest of this paper is organized as follows: Section 2 is about the state of the art where we give brief definition of some existing buffer management policies and other characteristics of DTNs. Section 3 describes our proposed algorithm. Section 4 summarizes performance metrics and shows simulation results and discussion. And finally, Section 6 is reserved for conclusion and future work.

\section{State of the Art}

\section{A. Existing Buffer management policies (Drop policies)}

In order to deal with different problems related to buffer overload, researchers have proposed a set of drop policies which give different results depending on the environment (traffic density, area size, buffer size, TTL ...). TABLE 1 shows a brief definition of some existing drop policies:

TABLE I

DESCRIPTION OF SOME BUFFER MANAGEMENT POLICIES

Policy Description

Drop Front Using this policy, the message which arrived first in the (FIFO) buffer will be selected first to be dropped. [3]

Drop Tail

(LIFO)

Drop Oldest

(SHLI)

E-Drop

(Equal Drop)

Drop Youngest

Drop

Largest

Evict Most

Forwarded

First (MOFO)

Evict Most

Favorably

Forwarded

First (MOPR)
The buffer in this strategy is ordered in a LIFO manner, so the message which arrives last to the queue will be the first dropped message

Every message has a time to live value (TTL), the message with the lowest TTL value is the oldest one, and is the first selected message to drop.

If there is no space to host the new coming message in the buffer, this policy deletes the message having the exact or the nearest size. This strategy minimizes the number of dropped messages. [4]

The youngest message is the message with the longest remaining time to live (TTL), and it is the first dropped message when this strategy is used. [5]

Each message is defined by a size; this buffer management strategy drops the message with the biggest size in the queue to free more space by dropping few messages. [6]

The message that has been forwarded to maximum number of nodes will be dropped first.[7]

Each message in node has a forwarding predictability $\mathrm{FP}$, initially set to 0 . When the message is forwarded, the FP value increases. The message with maximum FP value will be dropped first. [8] 


\section{B. Routing in DTN}

Routing protocols in Delay Tolerant Networks have many characteristics, so they can have many classifications. Some can classify routing algorithms as replication-based algorithms (i.e:the protocol creates message replicas) or forwarding-based (i.e: the protocol don't replicate messages) algorithms. [15]

Some DTN routing protocols can be classified in both categories like "spray and wait" witch benefits from the high delivery ratio of replication-based routing as well as the low resources consumption of forwarding-based routing, and which we will use in our simulation at the bottom of this paper.

NB: All routing protocols in Delay Tolerant Networks are based on Store-carry and Forward mechanism.

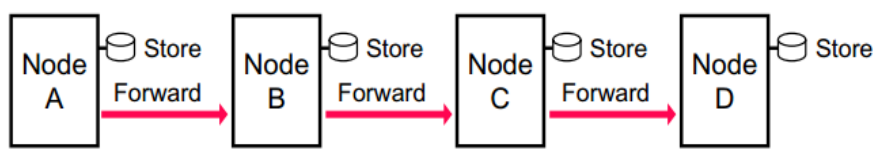

\section{1) FirstContact:}

A very simple routing algorithm for DTN, and a very quick one, this protocol forwards just a single copy of the message or the fragment of message to the first available contact.

\section{2) Epidemic}

From Wikipedia, an epidemic is the rapid spread of infectious disease to a large number of people in a given population within a short period of time. DTN researcher took the same idea to create a routing protocol where every node transmits replicas of its messages to newly discovered node which don't have it already. Theoretically, this algorithm needs to have unlimited buffer size and unlimited energy to give high rate of delivery, but practically this conditions are impossible to implement. [9]

\section{3) Prophet V2}

The Prophet routing protocol "probabilistic routing protocol using history of encounters and transitivity." operates on the assumption that human mobility is non-random, and that knowledge of the history of previous encounters is a good indicator of future encounters. In June 2011, Samo Grasic(samo@grasic.net) have proposed a new version of this protocol ProphetV2 white considers also the elapsed time during nodes encounters. [10]

\section{4) Spray \& Wait}

Spray and Wait combines the speed of epidemic routing with the simplicity of direct transmission. So, for the spray phase: for each message $M$ at the source node, $L$ copies of $M$ are forwarded to $L$ different relays (intermediate nodes). Then in the wait phase: if the destination is not found among the relays, every relay will perform direct transmission to the message destination only, and so on until each message in the source node buffer reaches its destination. [11]

\section{Nodes mobility in DTN}

Researchers have proposed many mobility models for DTN to track nodes geographic movement in the network by collecting a set of parameters like speed, direction, and acceleration of the node. In DTN there are many types of mobility models:

\section{1) Analytical models}

Analytical mobility models are based on mathematic equations for the prediction of the next position of each node

\section{a) Random walk}

For this model, the parameters used to determine the next position of the node are the direction and the movement speed. First, the node choose a direction angle between 0 and $2 \pi$, then it chooses a random speed between [Vmin, Vmax] and finally after reaching $t$ time it chooses other random values starting from this new position to another.

\section{b) Random waypoint}

By the opposite of the precedent model, in this one the node stops for a random pause period when it reaches a destination point before it chooses other speed and direction angle.

\section{2) Traces based models}

Traces based model are more realistic mobility models than analytical models, and are obtained from real results. These results are more accurate where the number of mobile nodes is important. The resulted traces are used then to make a new mobility model or to improve existing ones. Some examples are discussed here:

\section{a) ZebaNet}

A model made by collected information from some electronic devices placed on zebras at SweetWaters natural reserve in Kenya. [12]

\section{b) DakNet}

This model is implemented in developing countries, where the cost of implementing an infrastructure is very high. For this reason the messages are routed by bus or motorcycles traveling between villages. [12]

\section{Proposed Policy "MaxHopCount”}

\section{A. Description}

During its way to destination, every message traverses many nodes and stay a while in every node's buffer, before jumping to another node. Every message has a set of information, such as size, source, destination... etc. among these informations, there is "hop count", it refers to the number of nodes the message has crossed during its path from source to the current node. A high hop count means that the message crossed lot of node, and then there may be lot of copies at the network, so dropping this message from the buffer may not impact its delivery. Otherwise, a low hop count means low number of replicas at the network so dropping this message may lessen the delivery probability.

\section{B. The Flow-sheet:}

First, the function checks if the message size is greater than the whole buffer size, if so, the function ends because the message is too big. Otherwise, is initializes $m$ by the message with the highest Hop Count if $\mathrm{m}$ is null, so the function cannot delete any more messages and the new message can't be hosted at this node. If $m$ is not null we free the buffer from the message that $\mathrm{m}$ is pointing at, and we compare the size of the new coming message with the free buffer size. We repeat those steps until the buffer have enough space to host the new message. Fig. 1 below summarizes this algorithm 


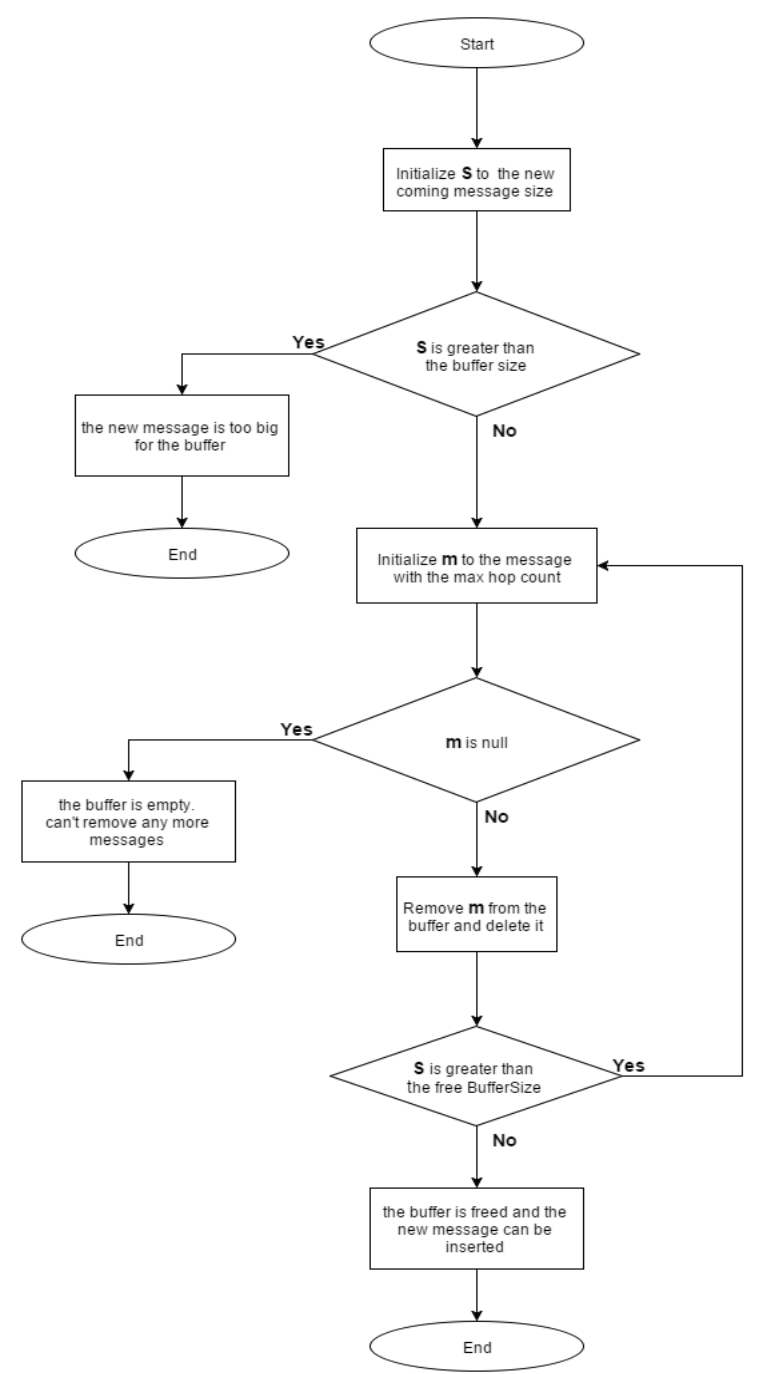

Fig. 1. The flow sheet of the new drop policy MaxHopCount

\section{RESUlTS AND DisCUSSION}

\section{A. The ONE simulator}

The ONE Simulator is a discrete event simulator written in Java. The major aspire of simulator is to relate DTN (store-carry-forward) of message for long time, where the probability of disconnections and failures enlarged. [13]

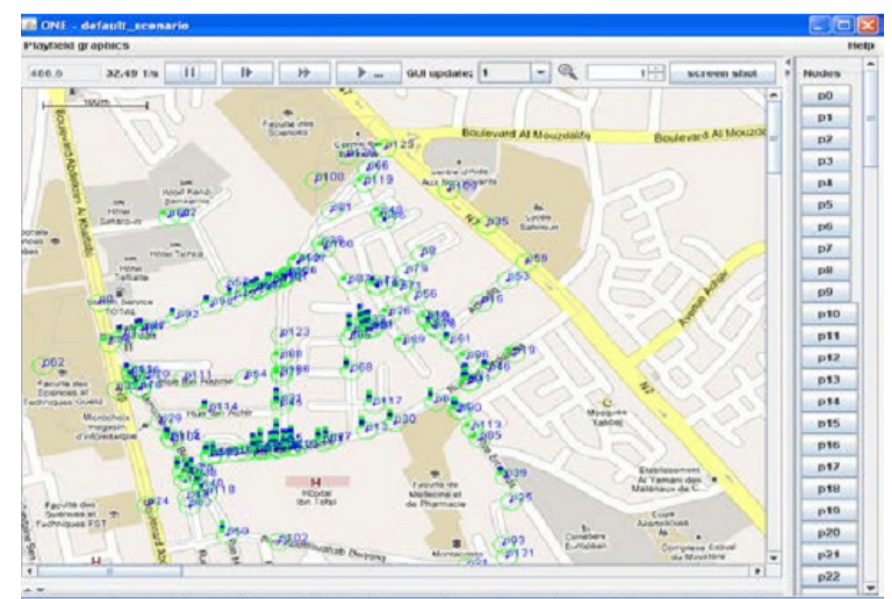

Fig. 2. ONE GUI mode of the ONE Simulator area: Helsinki city.

\section{B. Common metrics for performance evaluation}

The following metrics are commonly used when evaluating scenarios related to DTN protocols. [14]

\section{1) Delivery ratio.}

Suppose that $\mathrm{M}$ be the set of all messages created in the network and Md be the set of all messages delivered. Then, the delivery ratio is computed as:

$$
M_{d} / M
$$

\section{2) Average message delivery latency.}

Now let the $\mathrm{i}^{\text {th }}$ delivered message was created at time $\mathrm{c}_{\mathrm{i}}$ and delivered at time $d_{i}$. Then the average message delivery latency is computed as:

$$
\left(\sum_{i=1}^{M_{d}}\left(d_{i}-c_{i}\right)\right) / M_{d}
$$

\section{3) Overhead ratio}

Let $r_{i}$ be the number of replications of any message $m_{i} \in M$. Then the overhead ratio is determined as:

$$
\left(\sum_{i=1}^{M}\left(r_{i}-M_{d}\right)\right) / M_{d}
$$

\section{Simulation parameters}

To simulate our work we switch between different environments and we change many parameters such as routing protocols where we switch between Epidemic and ProphetV2 routers. Table II shows the important parameters of our simulations.

\begin{tabular}{|c|c|}
\hline Variable & Value \\
\hline movement Model & RandomWalk \\
\hline Router & Epidemic - ProphetV2 \\
\hline buffer Size & $10 \mathrm{M}$ \\
\hline drop Policy & $\begin{array}{l}\text { FIFO - LIFO - DL - DY - SHLI - } \\
\text { MaxHopCount - MOFO }\end{array}$ \\
\hline $\begin{array}{l}\text { Message TTL (in } \\
\text { minutes) }\end{array}$ & $60-120-180-240-300$ \\
\hline number of Hosts & 200 \\
\hline $\begin{array}{l}\text { Message creation } \\
\text { interval (in seconds) }\end{array}$ & 25,35 \\
\hline Messages size & $500 \mathrm{k}, 1 \mathrm{M}$ \\
\hline $\begin{array}{l}\text { World Size (width, } \\
\text { height; meters) }\end{array}$ & 500,500 \\
\hline
\end{tabular}

TABLE II

\section{SIMULATION PARAMETERS}

\section{Epedimic}

In the first simulation we compare different drop policies with different TTL values, then we analyse the results in term of delivery rate and overhead ratio. The routing protocol of this first simulation is Epidemic. 


\section{1) Delivery rate}

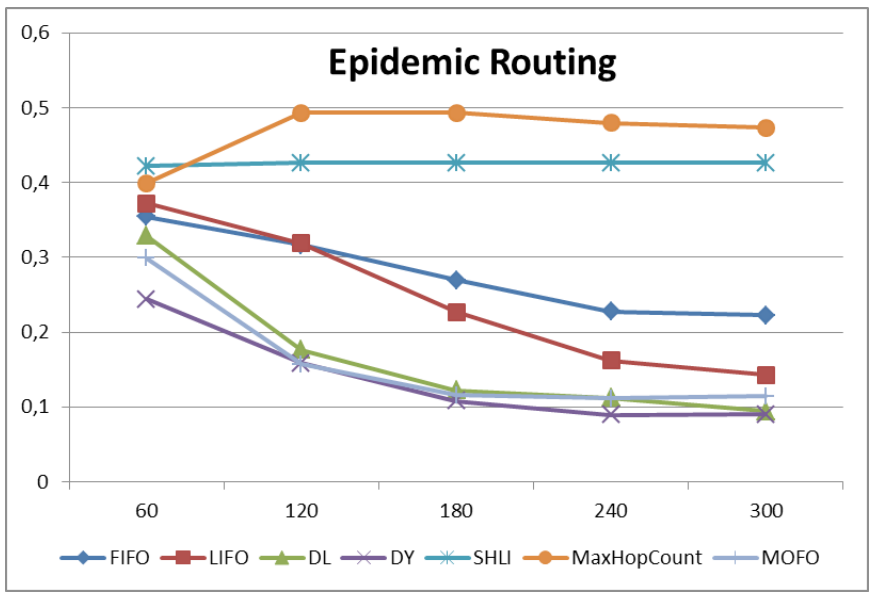

Fig. 3. Delivery rate as a function of TTL for different drop policies. The router is Epidemic.

As shown in the graph above, MaxHopCount policy, have a good delivery probability when the TTL value is high. the weakness of other policies, apart from SHLI (Shortest TTL), is that the delivery rate keep decreasing while the TTL value is heightening.

\section{2) Overhead Ratio}

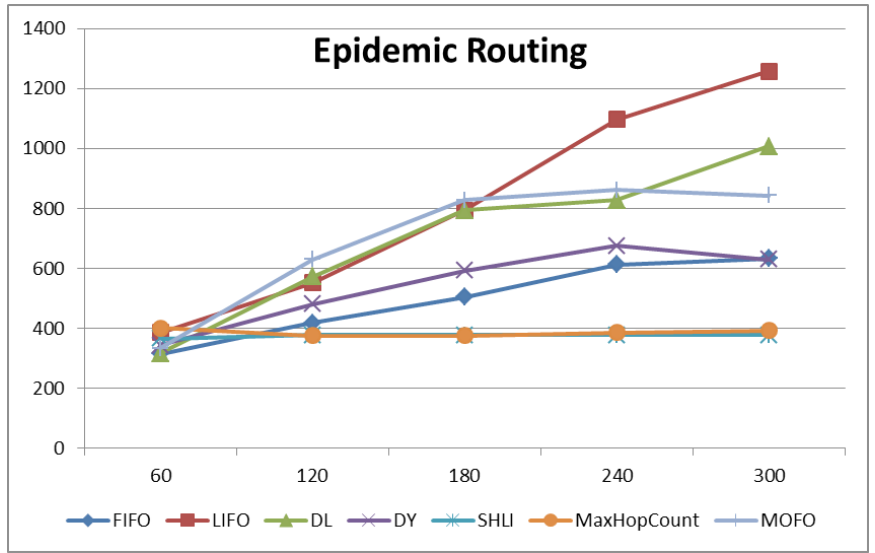

Fig. 4. Overhead ratio as a function of TTL for different drop policies. The router is Epidemic.

As shown above, the Overhead ratio is the number of message replicas in the network, so the main goal of all DTN algorithms is to minimize this ratio.

From Fig. 4, we observe that MaxHopCount policy doesn't overload the networks with message replicas because it gets low overhead ratio, this ratio keeps lessening which means that our new policy is also optimal in term of network overload.

\section{E. ProphetV2}

For the second simulation, the chosen routing protocol is ProphetV2. Like the first simulation, we compare here different drop policies with different TTL values, then we analyse the results in term of delivery rate and overhead ratio.

\section{1) Delivery rate}

In the opposite of Epidemic, ProphetV2 is an example of a probabilistic routing protocol. In this case, MaxHopCount have the highest delivery rate as well. And the increasing of the TTL value doesn't impact it.

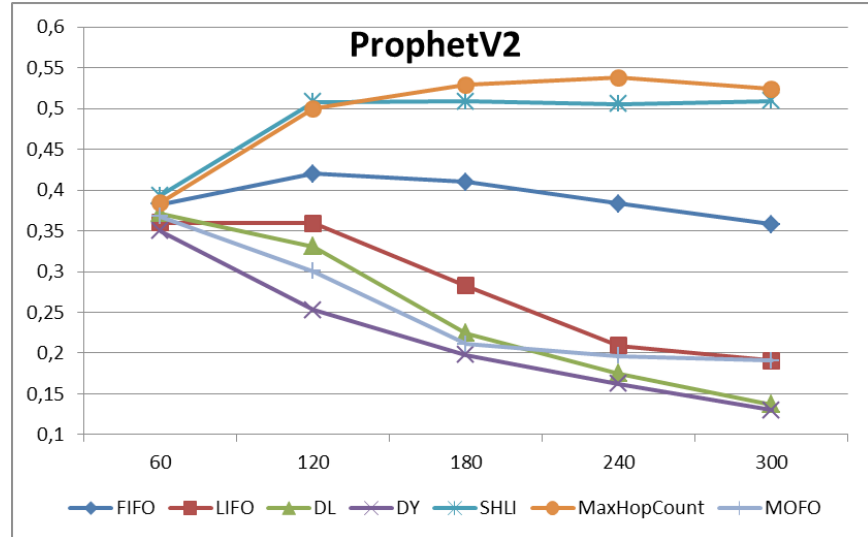

Fig. 5. Delivery rate as a function of TTL for different drop policies. The router here is ProphetV2.

\section{2) Overhead ratio}

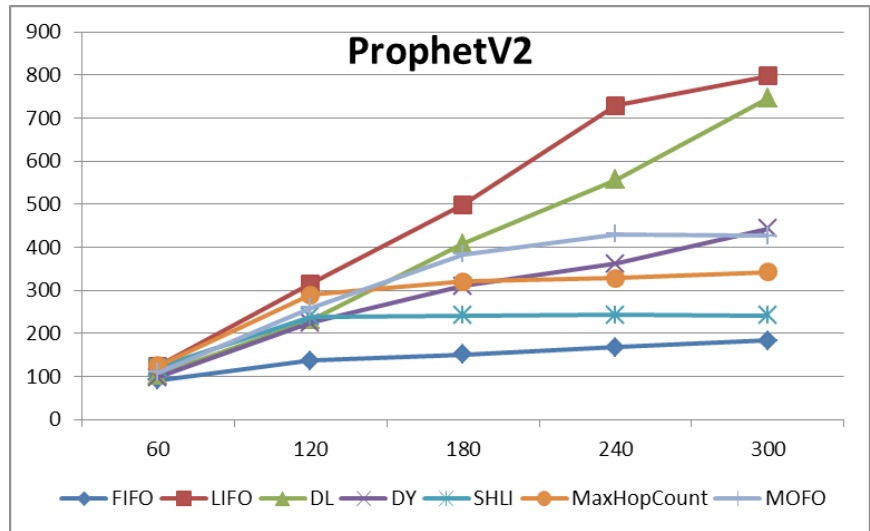

Fig. 6. Overhead ratio as a function of TTL for different drop policies. The router here is ProphetV2.

From Fig. 6 we can notice that the overhead ratio of our policy is not the lowest one, but it has a stable value and it is better than other policies where the high TTL have a very negative impact on this metric

\section{CONCLusion \& Future Works}

In this work, we compared some existing DTN drop policies to our new proposed algorithm, and we discussed the simulations results in term of delivery rate, overhead ratio, then we deduct that our new policy MaxHopCount is optimal for high TTL values (greater than 180 minutes or 3 hours), which is more realistic because usually in real scenarios the TTL may reach many days.

As a future work, we are working on this policy to study its efficiency with other parameter such as the area size, the message size... and we have also to choose the policy to be applied in ambiguous situations like equals numbers of hop.

\section{REFERENCES}

[1] Fall, K. (2003). A Delay Tolerant Networking Architecture for Challenged Internets. SIGCOMM.

[2] RFC 1155

[3] Sulma Rashid, Q. A., M. Soperi Mohd Zahid, A.Hanan. Abdullah (2011). "E-DROP: An Effective Drop Buffer Management Policy for DTN Routing Protocols." International Journal of Computer Applications 13(7): 8-13.

[4] Yun Li, L. Z., Zhanjun Liu, Qilie Liu (2009). "N-Drop: congestion control strategy under epidemic routing in DTN." IWCMC '09 Proceedings of the 
2009 International Conference on Wireless Communications and Mobile Computing: Connecting the World Wirelessly: 457-460

[5] Thrasyvoulos Spyropoulos, K. P., Cauligi S. Raghavendra (2005). Spray and Wait: An Efficient Routing Scheme for Intermittently Connected Mobile Networks. SIGCOMM'05 Workshops. Philadelphia, PA, USA.

[6] Sulma Rashid, Q. A., M. Soperi Mohd Zahid, A.Hanan. Abdullah (2011). "Impact of Mobility Models on DLA (Drop Largest) Optimized DTN Epidemic routing protocol." International Journal of Computer Applications 18: 35-39.

[7] Krifa, A. B., C. (2008). Optimal Buffer Management Policies for Delay Tolerant Networks. 5th Annual IEEE Communications Society Conference on Sensor, Mesh and Ad Hoc Communications and Networks. San Francisco, CA: 260 - 268.

[8] Samina Mansuri, H. S., Yogeshwar Kosta "Performance Analysis of Drop Policies for Different Mobility Models in DTN." nternational Journal of Computer Applications 59 (14).

[9] Amin Vahdat, D. B. "Epidemic Routing for Partially-Connected Ad Hoc Networks."

[10] Ahmed El Ouadrhiri, I. R., Mohamed El-Kamili, Ismail Berrada (2014). Controlling messages for probabilistic routing protocols in Delay-Tolerant Networks. ISCC: 1-6.

[11] Thrasyvoulos Spyropoulos, K. P., Cauligi S. Raghavendra (2005). Spray and Wait: An Efficient Routing Scheme for Intermittently Connected Mobile Networks. SIGCOMM'05 Workshops. Philadelphia, PA, USA.

[12] (2012). Une solution tolérante aux délais pour des applications de localisation et de traçabilité a posteriori en milieux confinés, UNIVERSITÉ BLAISE PASCAL.

[13] Ari Keränen, J. O., Teemu Kärkkäinen (2009). The ONE Simulator for DTN Protocol Evaluation. 2nd International Conference on Simulation Tools and Techniques. Rome, Italy.

[14] Saha, B. K. (2014). "Commonly Used Metrics for Performance Evaluation.” Retrieved 20 Sep 2014, 2014, from http://delay-tolerantnetworks.blogspot.com/2014/03/commonly-used-metrics.html.

[15] Torres-Franco, E., et al. "A Quantitative Justification to Dynamic Partial Replication of Web Contents through an Agent Architecture", International Journal of Interactive Multimedia and Artificial Intelligence, 3(3), pp. 82-88, 2015.

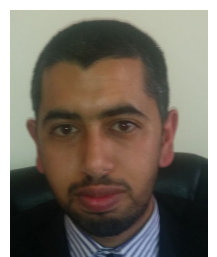

Youssef Harrati IT Engineer graduated in 2013 from the Cadi Ayyad University of Marrakech / Morocco. $\mathrm{PhD}$ student at the Laboratory of Applied Mathematics and Computer Science (LAMAI) of the doctoral studies center of Cadi ayyad University Marrakech / Morocco. His research interests are computer science, Delay Tolerant Networks, sensor networks and interplanetary networks.

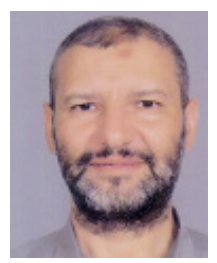

Abdelmounaïm Abdali $\mathrm{PhD}$ in Solid Mechanics and Structures in University of Amiens in 1996, France. He is a Professor in computer science at the University Cadi Ayyad, Faculty of Sciences and Technics, Marrakech, Morocco. Member at the Laboratory of Applied Mathematics and Computer Science (LAMAI) Marrakech,Morocco. His research interests are computer science, DTN Network, business intelligence, Semantic web, Big Data , ubiquitous computing, biomechanics, bone remodeling, numerical simulation in solid mechanics and structures. 